\title{
Manifestações iniciais de trocas interativas mãe-bebê e suas transformações
}

\author{
Adriana F. Paes Ribas \\ Maria Lucia Seidl de Moura \\ Universidade do Estado do Rio de Janeiro
}

\section{Resumo}

Neste trabalho, adota-se a perspectiva sócio-cultural e pressupõe-se que as interações adulto-bebê são matrizes nas quais se constrói o desenvolvimento. Esta pesquisa teve como objetivo descrever e analisar as transformações nas atividades da mãe e do bebê, nas trocas interativas e nos contextos específicos em que tais atividades e interações ocorrem, em etapas iniciais de desenvolvimento do bebê. Foi realizado o registro em vídeo, em ambiente natural, da observação de uma díade mãe-bebê em quatro momentos de desenvolvimento do bebê: 2, 10, 15 e 21 semanas. Foram analisadas as modificações na natureza das interações, mudanças nas atividades dos parceiros (e.g., vocalização, fala, sorriso), nos tipos de estimulação por parte das mães (e.g., estimulação voltada para a mãe ou voltada para os objeto) e os diferentes contextos de interação. Os resultados obtidos mostraram-se convergentes com achados de pesquisas na área, ampliando-os. Foram identificadas, desde fases iniciais, interações como processos recíprocos de engajamento que tornaram-se mais freqüentes e complexos. Foi possível identificar interações mãe-bebê, caracterizá-las e ilustrar a natureza diferenciada das atividades e interações dos parceiros em momentos distintos do desenvolvimento do bebê. Os resultados apresentados puderam ser interpretados segundo a abordagem sócio-cultural, que considera as interações sociais como constitutivas do desenvolvimento.

Palavras-Chave: Interação, interação mãebebê, desenvolvimento infantil inicial 
Key words: Interaction; mother-infant interaction; infant behavior

\begin{abstract}
Developmental changes in the patterns of mother-infant interaction

This study aims at investigating developmental changes in the patterns of mother-infant interaction, at early stages of the baby's development. A mother-infant dyad was observed and video recorded at home at four moments of the baby's development: $02,10,15$ and 21 weeks. The modifications in the nature of the interaction, in the partners' activities (e.g. vocalization, speech, smile), in the kinds of stimulation promoted by the mother (e.g. directed to herself or to objects), and in the different interactional contexts were examined. Results were consistent with the literature in the area and have expanded it. It was possible to identify interactions as reciprocal processes of engagement which became gradually more frequent and complex. Mother-infant interactions were identified since early stages. They were characterized and illustrated the differentiated nature of activities of the partners and interactions at distinct moments of the baby's development.
\end{abstract}

\section{$\mathrm{T}$}

em crescido o interesse pelo estudo psicológico da natureza e características da interação mãe-bebê, sendo já expressiva a literatura nesta área (e. g., Bornstein \& Tamis-LeMonda, 1990; Feldman, Greenbaum, Mayes \& Erlich, 1997). A partir dos anos sessenta, torna-se crescente o interesse por um modelo bidirecional, caracterizado pela ênfase na reciprocidade e na adaptação mútua entre os parceiros. Como diz Bruner (1996), sintetizando esta literatura, "o que esses estudos revelaram foi a importância da interação humana de mão dupla (p. 72)". Não se pensa em um bebê que apenas é estimulado pelas ações dos adultos, mas em trocas de parceiros de capacidades distintas.

Apesar desta mudança de enfoque e de um corpo de evidências empíricas em crescimento, nem sempre essas trocas interacionais têm sido analisadas e interpretadas em termos de seu papel no de- 
senvolvimento na ontogênese. Adotando-se a perspectiva que tem sido denominada por alguns autores como sócio-cultural (Bruner, 1996; Cole, 1998; Rogoff \& Chavajay, 1995), influenciada, entre outros e, principalmente, por Vygotsky $(1981,1984,1987)$, é possível pressupor que as interações adulto-bebê são matrizes através das quais se constrói o desenvolvimento. A atividade mediada é considerada, nesta abordagem, como unidade de análise dos processos psicológicos e as interações são fundamentais para a apropriação de ferramentas (artefatos) e sua internalização. Onde se inicia este processo? Pensa-se que nas primeiras interações dos bebês humanos com membros adultos de sua cultura, representados a princípio pela mãe. Deste modo, como discutido em Ribas e Seidl de Moura (1998), considera-se que a gênese de processos característicos do desenvolvimento cognitivo, tais como: conhecer o mundo, seus objetos e eventos, conhecer o outro, aprender a interagir, aprender a regular comportamentos, aprender a dar significado às situações, se dá nas interações mãe-bebê, desde fases precoces do desenvolvimento.

A literatura sobre interações iniciais mãe-bebê é expressiva. Têm sido investigados, entre outros aspectos, as modificações nas atividades dos parceiros (e.g., vocalização, fala, sorriso), nos tipos de estimulação por parte das mães - e.g., estimulação voltada para a mãe, estimulação voltada para os objetos do ambiente - (Bornstein \& Tamis-LeMonda, 1990; Cohn \& Tronick, 1987; Kaye \& Fogel, 1980), os tons de conversação utilizados pelas mães - e.g. tom infantil, tom adulto - (Grieser \& Kuhl, 1988) e os diferentes contextos de interação (Feldman et al., 1997).

Mudanças na quantidade e no tipo de estimulação por parte das mães com o aumento da idade do bebê têm sido observadas. Belsky, Gilstrap e Rovine (1984), observando bebês de 01, 03 e 09 meses, constataram um aumento significativo na quantidade de estimulação por parte das mães, que cada vez mais provocavam e chamavam a atenção dos bebês. Bornstein e Tamis-LeMonda (1990), pesquisando díades com bebês de 02 e 05 meses, observaram neste período um aumento do nível de estimulação didática, ou seja, voltada para os objetos, por parte das mães. 
O tom de conversação utilizado pela mãe também parece modificar-se ao longo do tempo. Grieser e Kuhl (1988) destacam o uso de um tipo de conversação especial dirigido aos bebês, que tem uma entonação especial e apresenta simplificação semântica e sintática denominado por alguns autores de motherese). Bornstein e TamisLeMonda (1990) observaram que quando as mães lidam com bebês aos dois meses de idade falam mais em motherese. Já aos cinco meses de idade do bebê modificam sua forma de falar, reduzindo o uso do tom infantil e aumentando o uso do tom de conversação adulto.

Em termos dos comportamentos dos bebês, tem sido destacada a mudança no interesse visual, na medida em que tende a diminuir a proporção de tempo que eles despendem olhando para a face de suas mães, entre 6 e 26 semanas, segundo dados de Kaye e Fogel (1980). Outro aspecto é o aumento gradual na freqüência de sorrisos e demonstrações de excitação e exploração por parte do bebê ao longo dos primeiros meses (Belsky et al., 1984).

Os evidências dessas pesquisas têm contribuído para a compreensão da natureza e das transformações das interações iniciais adulto-bebê e corroboram a idéia de influência e adaptação recíproca desses parceiros.

Este trabalho se alinha a essas iniciativas, buscando focalizar as transformações evolutivas dessas primeiras interações e trazer subsídios para a compreensão da gênese do processo de desenvolvimento constituído no contexto sócio-cultural através de interações. Considera-se que evidências de estudos brasileiros sobre este tema, atualmente ainda pouco freqüentes, podem ser interessantes nessa busca.

Seu objetivo é descrever e analisar as transformações nas atividades da mãe e do bebê, nas trocas interativas, e nos contextos específicos em que ocorrem, observadas em uma díade em quatro momentos de desenvolvimento do bebê: $2,10,15$ e 21 semanas.

\section{Método}

\section{Participantes}

Uma díade mãe-bebê (menino, nascido a termo e saudável) participou desta pesquisa. A mãe, com 27 anos na ocasião da coleta de 
dados, é de classe social média e nível de instrução superior completo. A idade do bebê na data da primeira observação era de 16 dias ( 2 semanas), na segunda 2 meses e 18 dias (10 semanas), na terceira 3 meses e 22 dias (15 semanas) e na quarta 5 meses e 11 dias (21 semanas). A díade residia em um bairro de classe média urbana da cidade do Rio de Janeiro.

\section{Procedimentos}

A díade mãe-bebê foi visitada quatro vezes em sua residência por um observador, em situações em que estavam apenas os dois. A mãe foi instruída a manter a sua rotina diária e a ignorar a presença do observador, na medida do possível. Foi realizado o registro em vídeo das atividades da mãe e do bebê durante 30 minutos, iniciado após 10 minutos de familiarização com o observador.

\section{Levantamento de Dados}

Dos trinta minutos de vídeo, realizados em cada uma das quatro observações, foram desconsiderados os primeiros cinco minutos por se considerar que representavam um tempo inicial de familiarização dos sujeitos com a situação de filmagem. Os vinte minutos seguintes foram analisados e descartou-se os últimos cinco minutos de observação. Os vinte minutos de vídeo considerados foram analisados em intervalos fixos de 30 segundos. Foi utilizado o método de registro por intervalos, registrando-se a ocorrência ou não de cada uma das categorias de observação nos intervalos (Fagundes, 1985).

Os dados foram inicialmente levantados através do preenchimento da Folha de Registro de Interação e Atividades (FRIA), uma planilha para registro desenvolvida especialmente para este estudo. Na FRIA foram registradas as ocorrências de atividades da mãe e do bebê, ocorrências de interação e de tentativas de interação não efetivadas, nos intervalos. Foram, ainda, descritos os contextos de troca em cada um dos intervalos. Foram transcritas todas as falas e vocalizações da mãe e do bebê durante as observações.

\section{Categorias de Observação}

A categorização proposta a seguir foi desenvolvida pelas autoras, com exceção das seguintes categorias: domínio social e domínio 
didático, propostas por Bornstein e Tamis LeMonda (1990) e Vibbert e Bornstein (1989).

Categorias de observação de interação: domínio social de interação (DS), domínio didático de interação (DD) e tentativa de interação não efetivada (T) (Bornstein \& Tamis-LeMonda, 1990; Vibbert \& Bornstein, 1989).

O domínio social (DS) de interação é identificado quando no episódio de interação a mãe usa estratégias físicas e/ou verbais para engajar a criança interpessoalmente, ou seja, para fazer com que o bebê volte a atenção para ela. E o bebê dirige comportamentos sociais em relação à mãe, se mostrando engajado interpessoalmente com ela.

O domínio didático (DD) é identificado quando a interação se volta para fora da díade, ou seja, quando a mãe usa estratégias físicas e/ou verbais para provocar (despertar) e estimular a criança a se engajar numa propriedade, evento ou objeto no ambiente fora da díade e quando o bebê tem um foco correspondente de atenção no ambiente.

A tentativa de interação não efetivada (T) é caracterizada por um dos parceiros dirigir a atividade em relação ao outro e o outro não dirigir nenhuma atividade como resposta.

Categorias de observação de atividade (da mãe e do bebê): gestos $(\mathrm{G})$, vocalizações $(\mathrm{V})$, fala $(\mathrm{F})$, atribuição de significado (AS), sorrir (S), toque de afeto (TA), toque de cuidado (TC), olhar o bebê (OB), mostrar objeto (MO), cantar (CA) e pegar no colo (PC). As categorias de observação de atividade do bebê são: olhar objeto $(\mathrm{OO})$, olhar o ambiente (OA), olhar a mãe (OM), movimentar membros (MM), tocar a mãe (TM), mamar (M), pegar objeto (PO), vocalizar (V), sorrir (S) e fechar os olhos (FO).

Categorias de observação de contexto: foram classificados os contextos específicos nos quais se davam as atividades, interações e tentativas por parte da díade, que, basicamente, foram os seguintes: cuidado (e.g., troca de fralda, banho), amamentação, bebê no colo da mãe (sem que o bebê estivesse mamando), bebê deitado na cama, com a mãe sentada ao lado dele e mãe com bebê no colo, estando o bebê com o rosto voltado de frente para ela. 


\section{Índice de Fidedignidade}

Foi calculado o índice de fidedignidade (IF= SA / S (A+D) $\mathrm{x}$ 100) relativo ao levantamento de dados, considerando-se os acordos (A) e desacordos (D) de ocorrência de cada categoria observada, obtidos entre juizes e observadores (Kadzin, 1982). Para tal, trechos dos vídeos, correspondendo a $20 \%$ do tempo total de observação, foram selecionados aleatoriamente e submetidos a dois juizes.

\section{Análise dos Dados}

A análise dos dados compreendeu os seguintes procedimentos: (a) análise das percentagens de ocorrência de cada categoria de observação (de atividades da mãe, do bebê, de interação e tentativa) em relação ao total de intervalos considerados; (b) análise qualitativa de episódios de interação e tentativa, ou seja, uma análise minuciosa das atividades realizadas pelos parceiros, do(s) contexto(s) no qual tais atividades ocorreram e a duração dos episódios e (c) análise das falas e vocalizações da mãe durante a observação, avaliando seu conteúdo e identificando atribuições de significado aos comportamentos do bebê. Após a análise particular de cada uma das observações, foram realizadas comparações entre os quatro períodos observados, buscando-se investigar as transformações na natureza das atividades, interações e tentativas de interação da díade.

\section{Resultados e Discussão}

Os índices de fidedignidade obtidos para os grupos de categorias de observação foram os seguintes: Categorias de observação de Interação - IF $=74 \%$; Categorias de observação de Atividade do Bebê - IF $=75 \%$; Categorias de observação de Atividade da Mãe - IF $=73 \%$.

A Tabela 1 mostra as percentagens de ocorrência de atividades e interações nas quatro observações.

As atividades do bebê concentraram-se inicialmente em: olhar a mãe, fechar os olhos e mamar e as atividades com maior percentagem de ocorrência, por parte da mãe foram: olhar o bebê e tocá-lo. Na observação realizada quando o bebê estava com 10 semanas a mãe passa a utilizar uma maior variedade de comportamentos para 
280 A. F. P. Ribas e M. L. Seidl de Moura

Tabela 1

Percentagens de ocorrência de atividades e interações

\begin{tabular}{|c|c|c|c|c|c|}
\hline & & \multicolumn{4}{|c|}{ Observação } \\
\hline & & 01 & 02 & 03 & 04 \\
\hline \multirow{4}{*}{ Interação } & Interação & 17,5 & 37,5 & 80,0 & 52,5 \\
\hline & Domínio Social & 17,5 & 22,5 & 47,5 & 20,0 \\
\hline & Domínio Didático & 0,0 & 2,5 & 0,0 & 12,5 \\
\hline & $\begin{array}{l}\text { Domínio } \\
\text { Social/Didático }\end{array}$ & 0,0 & 12,5 & 32,5 & 20,0 \\
\hline \multirow{11}{*}{$\begin{array}{l}\text { Atividades } \\
\text { da Mãe }\end{array}$} & Gesto & 0,0 & 12,5 & 22,5 & 12,5 \\
\hline & Vocalização & 0,0 & 5,0 & 27,5 & 7,5 \\
\hline & Fala & 7,5 & 95,0 & 100,0 & 85,0 \\
\hline & Sorriso & 7,5 & 52,5 & 80,0 & 67,5 \\
\hline & Toque de Afeto & 32,5 & 57,5 & 75,0 & 52,5 \\
\hline & Toque de Cuidado & 7,5 & 15,0 & 12,5 & 17,5 \\
\hline & Olhar bebê & 100,0 & 100,0 & 100,0 & 100,0 \\
\hline & Mostrar Objeto & 0,0 & 20,0 & 30,0 & 37,5 \\
\hline & Cantar & 0,0 & 2,5 & 2,5 & 0,0 \\
\hline & Pegar no Colo & 5,0 & 0,0 & 10,0 & 0,0 \\
\hline & Atribuir Significado & 2,5 & 20,0 & 40,0 & 35,0 \\
\hline \multirow{10}{*}{$\begin{array}{c}\text { Atividades } \\
\text { do Bebê }\end{array}$} & Olhar Objeto & 0,0 & 20,0 & 47,5 & 40,0 \\
\hline & Olhar Ambiente & 7,5 & 67,5 & 72,5 & 80,0 \\
\hline & Olhar Mãe & 60,0 & 47,5 & 88,0 & 42,5 \\
\hline & $\begin{array}{l}\text { Movimentar } \\
\text { Membros }\end{array}$ & 7,5 & 95,0 & 100,0 & 90,0 \\
\hline & Tocar Mãe & 7,5 & 2,5 & 0,0 & 10,0 \\
\hline & Mamar & 82,5 & 10,0 & 0,0 & 20,0 \\
\hline & Pegar Objeto & 0,0 & 0,0 & 0,0 & 0,0 \\
\hline & Vocalizar & 0,0 & 37,5 & 62,5 & 22,5 \\
\hline & Sorrir & 10,0 & 32,5 & 57,5 & 40,0 \\
\hline & Fechar Olhos & 87,5 & 0,0 & 0,0 & 20,0 \\
\hline
\end{tabular}


se relacionar com o bebê, incluindo atividades como: falar, sorrir e mostrar objeto. Estes dados sugerem que ocorre, por parte da mãe, um ajuste no tipo de atividade que utiliza para comunicar-se e interagir com o bebê. O repertório de comportamentos do bebê se amplia de forma bastante significativa. Aumentam a exploração do ambiente, o interesse por olhar objetos e a atividade motora. A atividade de olhar a mãe aparece, ainda, como uma forma importante de comunicação usada pelo bebê, sendo associada ao sorriso e à vocalização. Na observação realizada quando o bebê estava com 15 semanas ocorre, por parte da mãe, uma percentagem maior de sorrisos, vocalizações e o aumento da atividade de mostrar objetos para o bebê. As modificações nas atividades do bebê mostram-se relacionadas às que ocorreram nas atividades da mãe, na medida em que houve, igualmente, por parte do bebê, um aumento na percentagem de sorrisos, vocalizações e na atividade de olhar para objetos. Na observação realizada quando o bebê estava com 21 semanas o repertório de atividades da mãe e do bebê é bastante semelhante ao da observação anterior, ocorrendo, entretanto, uma diminuição da atividade do bebê de olhar para a mãe e um aumento da atividade de olhar para o ambiente. Estes dados são consistentes com os achados de Bornstein e Tamis-LeMonda (1990) relativamente à frequiência de exploração do ambiente em bebês da faixa etária de cinco meses.

A Figura 1 mostra as variações nas percentagens de ocorrência de vocalização, sorriso, mostrar objeto e olhar objeto, por parte da mãe e do bebê, nas quatro observações e as curvas parecem indicar uma relação entre estas atividades.

Estes resultados sugerem que as atividades dos parceiros não devem ser consideradas apenas enquanto atividades individuais isoladas e, sim, como atividades que ocorrem na presença do outro e cuja ocorrência está sintonizada, vinculada aos comportamentos do parceiro. Observa-se, corroborando resultados de Brazelton e Cramer (1992), que as atividades dos parceiros, entre elas o sorriso e a vocalização, ocorrem num contexto, juntamente com outras atividades e tendem a estimular a produção, por parte do parceiro, de uma resposta correspondente. 


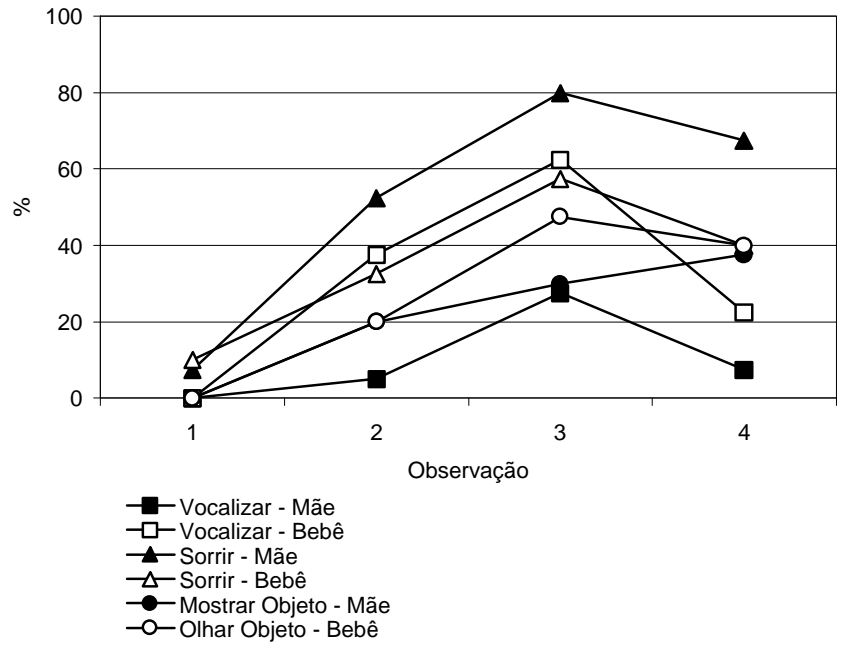

Figura 1

Atividades relacionadas mãe-bebê

As transformações nas atividades dos parceiros mostram o quanto a díade tende a, gradativamente, incorporar o mundo externo em suas interações. $\mathrm{O}$ bebê se interessa mais por eventos e objetos do ambiente e a mãe, talvez por perceber este interesse do bebê e atribuir a ele progressivamente uma maior capacidade de compreensão do que se passa a sua volta, tende mais freqüentemente a apresentar a ele tais objetos e eventos. Bornstein e Tamis LeMonda (1990) assinalam que tende a haver uma correspondência entre as atividades dos parceiros. O encorajamento social por parte da mãe está associado com uma orientação social por parte da criança. $\mathrm{O}$ mesmo se dá com o domínio didático.

Os episódios de interação mostraram uma tendência geral ao aumento da freqüência e sua natureza parece ter se modificado, uma vez que se transformaram seus domínios e sua complexidade. $\mathrm{Na}$ primeira observação foram identificados quatro episódios de interação, com uma duração média de 22,25 segundos. Todos os episódios foram de domínio social e se caracterizaram basicamente pelo 
olhar, o que pode ser considerado esperável, em função da idade do bebê. Como relatam Kaye e Fogel (1980), o contexto de trocas facea-face é bastante característico nos primeiros momentos do desenvolvimento.

Seqüências interativas mais complexas caracterizaram a segunda observação, na medida em que envolveram uma maior variedade de atividades por parte dos parceiros. As modalidades de comunicação que inicialmente restringiam-se a olhar e tocar, se ampliam e incluem olhar, tocar, sorrir, vocalizar, movimentar membros, mostrar e olhar para objetos. Foram identificados seis episódios de interação, dos quais, em quatro, aparece o domínio didático, com duração média de 61 segundos. Observou-se ainda um aumento da exploração do ambiente por parte do bebê e um aumento na iniciativa da mãe de estimulação didática. Estes achados convergem com as conclusões apresentadas por Bornstein e Tamis-LeMonda (1990), que relatam, por um lado, um aumento progressivo por parte do bebê, de exploração do ambiente e, por parte da mãe, de estimulação didática e, por outro, um aumento significativo da ocorrência de domínios didáticos de interação aos dois meses de idade.

Na terceira observação ocorreu um aumento expressivo do número de interações, sendo identificados dezesseis episódios, dos quais, seis de domínio didático, com duração média de 37,2 segundos. Foi crescente a diversidade de atividades da díade e a capacidade dos parceiros de regular seus comportamentos um em função do outro. Um aspecto descrito por Kaye e Fogel (1980) que pôde ser observado foi a "estratégia" utilizada pela mãe para atrair a atenção do bebê, apresentando objetos à ele e, em seguida, tentando desviar a atenção do bebê para ela própria.

Progressivamente, os parceiros alcançam um maior conhecimento recíproco, das formas de interagir e das possibilidades de regular mutuamente seus comportamentos. Como consequiência, a díade incorpora, cada vez mais, situações novas às suas interações. O número de episódios de interação identificados na última observação foi onze, dos quais seis de domínio didático, com duração média de 36 segundos. 
Considerando todo o período de observação, a duração média das interações foi de 40 segundos, variando entre 20 e 60 segundos. As diferenças nas durações dos episódios, observadas entre os períodos de observação, não foram testadas estatisticamente, mas não parecem ser significativas. Esses resultados sugerem que são principalmente as características do bebê como parceiro que impõem limites temporais à interação em todo o período inicial aqui estudado.

Como observam Feldman e colaboradores (1997), os contextos mais apropriados para as interações se transformam em diferentes estágios do desenvolvimento. Neste estudo, o contexto de troca identificado na primeira observação, na qual o bebê estava com duas semanas, foi: bebê no colo da mãe (em alguns momentos mamando e em outros não), que pode ser considerado como um contexto mais limitado e favorecedor de trocas face-a-face. Nas observações seguintes os contextos de troca modificaram-se e incluíram cada vez mais situações de jogo, manipulação de objetos e exploração do ambiente. Os contextos predominantes foram: a) bebê deitado na cama, estando a mãe sentada no chão ao lado da cama e b) mãe com bebê no colo, estando o bebê voltado com o rosto e o corpo de frente para ela.

A Tabela 2 resume as ocorrências de interação e tentativas de interação não efetivadas, nos quatro períodos de observação. A coluna " $F$ " indica a freqüência de episódios identificados. A coluna "\%" indica a percentagem de intervalos em que cada categoria ocorreu. A coluna "S/Tent S/Int" indica a percentagem de intervalos em que não foram registradas ocorrências de tentativa nem de interação.

Cada vez se observam menos intervalos em que os parceiros não interagem ou não tentam interagir. Além disso, observou-se que as tentativas de interação apareceram progressivamente mais encadeadas com os episódios de interação e funcionaram como uma espécie de preparação do contexto para a interação seguinte. Nas tentativas observadas, a mãe utiliza variados comportamentos com o objetivo de chamar a atenção do bebê. Entre eles, incluem-se: chamar o nome do bebê, vocalizar, propor brincadeiras, sorrir, acariciar, mostrar objeto e posicionar o rosto diante do rosto do bebê. 
Tabela 2

Ocorrências de Interação e Tentativa

\begin{tabular}{|c|r|r|r|r|r|}
\cline { 2 - 6 } \multicolumn{1}{c|}{} & \multicolumn{2}{c|}{ Tentativa } & \multicolumn{2}{c|}{ Interação } & \multicolumn{1}{c|}{$\begin{array}{c}\text { S/ Tent. } \\
\text { S/ Int. }\end{array}$} \\
\hline Obs. & \multicolumn{1}{c|}{ F } & \multicolumn{1}{c|}{$\%$} & F & \multicolumn{1}{c|}{$\%$} & \multicolumn{1}{c|}{$\%$} \\
\hline 1 & 01 & 2,5 & 04 & 17,5 & 80,0 \\
\hline 2 & 07 & 30,0 & 06 & 37,5 & 32,5 \\
\hline 3 & 06 & 17,5 & 16 & 80,0 & 2,5 \\
\hline 4 & 06 & 20,0 & 11 & 52,5 & 27,5 \\
\hline
\end{tabular}

A partir da segunda observação a atividade "falar" apresentou uma das maiores percentagens de ocorrência. O estilo de fala da mãe pareceu mais simplificado, em termos semânticos e sintáticos, nas observações 2 e 3 e um pouco mais complexo na observação 4 . $\mathrm{O}$ número de ocorrências desta atividade na primeira observação foi muito pequeno, o que não permitiu que se fizesse qualquer tentativa de caracterização da fala materna. O tom de voz usado pela mãe pareceu diferenciado, quando comparado ao tom de voz usado na fala com outro adulto.

Observou-se uma tendência crescente, por parte da mãe, de atribuir significados e/ou intenções aos comportamentos do bebê, especialmente a partir da segunda observação. A natureza das atribuições feitas pela mãe mostrou-se predominantemente vinculada a estados emocionais, preferências, competências do bebê (e.g., a mãe atribui ao bebê: querer, preferir, gostar mais de certas atividades do que de outras, estar calmo, estar nervoso) e não a necessidades fisiológicas (e.g., estar com fome, com sono). A natureza das atribuições pode ser interpretada como revelando uma concepção, por parte da mãe, de que o bebê, desde muito cedo, com 2 meses e 18 dias, como 
no caso da observação 2 , mostra-se competente sensorialmente e capaz de ter uma série de preferências e estados emocionais.

\section{Conclusões}

Tendo sido feita a observação de apenas uma díade, certamente os dados obtidos não refletem todas as situações de troca possíveis, mas mostram a possibilidade de caracterização de interações desde fases iniciais e sugerem que as atividades e interações dos parceiros podem ter natureza diferenciada em momentos distintos do desenvolvimento.

Os resultados obtidos forneceram informações sobre aspectos relacionados às transformações nas interações mãe-bebê e mostraram-se convergentes com achados de pesquisas na área (e.g., Bornstein \& Tamis-LeMonda, 1990), ampliando-os.

Foi possível identificar interações como processos recíprocos de engajamento que tornaram-se progressivamente mais complexos na medida em que os parceiros avançavam no conhecimento um do outro, acerca das interações e aperfeiçoavam a capacidade de ajuste e regulação mútua dos seus comportamentos.

Os resultados discutidos anteriormente podem ser interpretados segundo a abordagem sócio-cultural, que considera as interações sociais como constitutivas do desenvolvimento. As interações que ocorrem nas primeiras semanas de vida do bebê, representam, segundo esta abordagem, oportunidades de conhecer o mundo, seus objetos e eventos e também os parceiros e suas características. Pode-se dizer que é no contexto destas interações que o bebê se desenvolve.

\section{Referências}

Belsky, J., Gilstrap, B., \& Rovine, M. (1984). The Pennsylvania infant and family project I: stability and change in mother-infant and father-infant interaction in a family setting at one, three, and nine months. Child Development, 55, 692-705.

Bornstein, M., \& Tamis-LeMonda, C. S. (1990). Activities and interactions of mothers and their firstborn infants in the first six months of life: covariation, stability, continuity, correspondence and prediction. Child Development, 61, 1206-1217. 
Brazelton, T. B., \& Cramer, B. G. (1992). As primeiras relações. São Paulo: Martins Fontes.

Bruner, J. (1996). The culture of education. Cambridge: Harvard University Press.

Cohn, J. F., \& Tronick, E. Z. (1987). Mother-infant face-to-face interaction: the sequence of dyadic states at 3,6, and 9 months. Developmental Psychology, 23, 68-77.

Cole, M. (1998). Cultural psychology: a once and future discipline. Cambridge: The Belknap Press of Harvard University Press.

Fagundes, A. J. (1985). Descrição, definição e registro do comportamento. São Paulo: Edicon.

Feldman, R., Greenbaum, C. W., Mayes, L. C., \& Erlich, S. H. (1997). Change in mother-infant interactive behavior: relations to change in the mother, the infant, and the social context. Infant Behavior and Development, 20, 151-163.

Grieser, D. L., \& Kuhl, P. K. (1988). Maternal speech to infants in a tonal language: support for universal prosodic features in motherese. Developmental Psychology, 24, 14-20.

Kadzin, A. (1982). Single-case research designs: methods for clinical and applied settings. New York: University Press.

Kaye, K., \& Fogel, A. (1980). The temporal structure of face-to-face communication between mothers and infants. Developmental Psychology, 16, 454-464.

Ribas, A. F. P., \& Seidl de Moura, M. L. (1998). Interações precoces mãebebê: a gênese de zonas de construção. Cadernos de Psicologia, 9, 5066.

Rogoff, B., \& Chavajay, P. (1995). What's become of research on the cultural basis of cognitive development. American Psychologist, 50, 859877.

Vibbert, M., \& Bornstein, M. H. (1989). Specific associations between domains of mother-child interaction and toddler referential language and pretense play. Infant Behavior and Development, 12, 163-184.

Vygotsky, L. S. (1981). Thought and language. Cambridge: M. I. T. Press.

Vygotsky, L. S. (1984). A formação social da mente. Rio de Janeiro: Martins Fontes.

Vygotsky, L. S. (1987). The collected works of Lev Semenovich Vygotsky (vol. $1 \&$ 2). New York: Plenum.

Adriana F. Paes Ribas, psicóloga e mestre em Psicologia, é Bolsista de Apoio Técnico (FAPERJ). Maria Lucia Seidl de Moura, doutora em Psicologia Cognitiva 
288 A. F. P. Ribas e M. L. Seidl de Moura

pela Fundação Getúlio Vargas, é professora e Coordenadora do Programa de Pós-Graduação em Psicologia Social da Universidade do Estado do Rio de Janeiro.

Endereços para correspondência: Adriana F. P. Ribas, Email: aribas@ax.apc.org. Maria Lúcia Seidl de Moura, Rua Fritz Feigl, 465, Eldorado, Rio de Janeiro, Brasil. E-mail: mlseidl@ax.apc.org. 